\title{
A Survey on Energy Efficient Routing Protocols in Mobile Ad hoc Network
}

\author{
Neha Yadav ${ }^{1}$, Pooja Kundu ${ }^{2}$ \\ Assistant Professor, ECE Dept, St. Andrew Institute of Engineering and Management, Gurgaon ${ }^{1}$
}

Assistant Professor, CSE Dept, PIET College, Panipat ${ }^{2}$

\begin{abstract}
Mobile ad hoc networks (MANETs) are highly dynamic in nature. They are used in various critical and urgent situations such as in a conference rooms, virtual lecture rooms, and communication in case of disaster. They do not have a fixed infrastructure and nodes have limited power and bandwidth. Energy aware routing protocols are required for routing packets and choosing an optimal path in terms of energy and bandwidth. This paper presents a literature survey of such energy aware routing protocols. These protocols are modified from existing routing protocols.
\end{abstract}

Keywords: Routing Protocols, MANETs, Energy efficient, DSR, AODV.

\section{INTRODUCTION}

Wireless networks can be broadly classified into two categories namely infrastructure based networks and infrastructure less networks. Mobile ad hoc networks (Manet) come under the category of infrastructure less networks because they do not have a fixed base station for communication [1]. Nodes in a Manet act as routers and are responsible for sending and receiving data with the help of intermediate nodes. Nodes cooperate with each other to perform networking operations. Manet is getting importance because of its characteristics such as selfdeploying ability, dynamic topology, and easy deployment. They are preferable for critical conditions as in case of a natural calamity, military communication.

Besides several advantages Manets have several limitations. Nodes have limited battery-power. An efficient routing protocol is required because of dynamic topology of the network. Several routing protocols like DSR, AODV, etc. are used for routing the data packet from source to destination. Routing protocols are divided into three categories- reactive, proactive and hybrid. This paper presents several routing protocols for Manets and gives a comparative study of routing protocols. Major concern of this paper is to discuss energy efficient routing protocols.

This rest of the paper is divided into 4 sections. Section 2 describes different categories of routing protocols for Manet and challenges faced by routing protocols. Section 3 is focused towards giving a brief study of several energy efficient routing protocols. A tabularized comparative study of routing protocols for Manets is presented in section 4. Section 5 concludes the research.

\section{ROUTING PROTOCOLS FOR MANETS}

\subsection{Categories of Routing Protocols}

Routing protocols are categorized into three classes namely reactive, proactive and hybrid. Reactive routing protocols discover and establish routes only when there is a demand by the source node. Therefore, they are also known as on-demand routing protocols and because of this reason they have high latency. They do not require periodic updates of routing paths stored in tables at each node. So they have the advantage of having lesser overhead as compared to the pro-active routing protocols. They maintain information for only those routes which are active currently. Examples of routing protocols are DSR (Dynamic Source Routing), AODV (Ad hoc On-Demand Distance Vector Routing), etc.

In proactive routing protocols such as DSDV (Distance Sequence Distance Vector), OLSR (Optimized Link State Routing Protocol), every node updates the table containing route information periodically by monitoring the network. When a node wants to send a data packet to other node, the path is already known. So there is lesser latency in comparison to routing protocols. Periodic updates in MANETs results into overhead because nodes are dynamic in nature.

Hybrid routing protocols have the advantages of reactive and proactive routing protocols. They combine the features of reactive and proactive routing protocols. Mostly they act as proactive routing protocols initially, in order to have knowledge about the network and routes. Later on, they act as reactive routing protocols. Examples of such protocols are TORA (Temporally Ordered Routing Algorithm), ZRP (Zone Routing Protocol), ZHLS (zone based hierarchical link state routing protocol), etc.

Routing protocols for MANET can also be categorized by considering whether they are source-driven routing protocols or hop-by-hop routing protocol. Routing protocols which are source-driven broadcasts a packet or hello message for initiating routing. The latter category of protocols is deprived of the complete route information. 
Vol. 5, Issue 12, December 2016

So, they relay the packet hop-by-hop from one node to the other node in the network without having an actual knowledge of the destination.

Another way of categorizing the routing protocols can be single-path routing and multiple-path routing. In multiplepath routing, several routes are chosen to the destination and in this way load is divided among the nodes. Singlepath routing has a risk of failure of one or more nodes in the path and in that case alternative path has to be chosen. This consumes time and battery is wasted.

Routes can be discovered by using the information globally. For example, DSR discovers route to the destination by broadcasting the request packet. In large networks, broadcasting and re-broadcasting can result in flooding and overhead of control packets. Routes can also be discovered by using the information present at the nodes locally. Each node is aware of its neighborhood and this knowledge can be used to find the route. Table 1 summarizes all these categories of routing protocols with example.

\subsection{Challenges Faced by Routing Protocols for MANETs}

Each node in a MANET has a battery for transmitting and receiving data and control packets. These batteries have limited power. It is possible that a power source for charging the battery is not available or battery is not rechargeable especially in case of remote areas like war field. When a node has limited power or is dead then it starts dropping data packets. Bandwidth is also limited. Topology of these networks keeps changing dynamically. So it is very difficult for routing protocols to resolve all these issues. [2] The constraint of energy in MANETs results in need for energy efficient techniques in order to prolong the lifetime of MANET. Routing protocols for MANET can also be categorized by considering whether they are source-driven routing protocols or hop-by-hop routing protocol. Routing protocols which are sourcedriven broadcasts a packet or hello message for initiating routing. The latter category of protocols is deprived of the complete route information. So, they relay the packet hopby-hop from one node to the other node in the network without having an actual knowledge of the destination.

Another way of categorizing the routing protocols can be single-path routing and multiple-path routing. In multiplepath routing, several routes are chosen to the destination and in this way load is divided among the nodes. Singlepath routing has a risk of failure of one or more nodes in the path and in that case alternative path has to be chosen. This consumes time and battery is wasted.

Routes can be discovered by using the information globally. For example, DSR discovers route to the destination by broadcasting the request packet. In large networks, broadcasting and re-broadcasting can result in flooding and overhead of control packets. Routes can also be discovered by using the information present at the nodes locally. Each node is aware of its neighborhood and this knowledge can be used to find the route. Table 1 summarizes all these categories of routing protocols with example.

\subsection{Approach to Conserve Energy by Routing Protocols \\ Routing Protocols for MANETs can conserve energy in a network by using following approaches:}

- Load in a network can be balanced. Multiple paths can be chosen to route the packet to the destination. If a route gets damaged, then there is always another route available. So, delay can be reduced and bandwidth can be conserved in finding an alternative route.

- Nodes with limited residual energy and nodes with high residual energy can work together to relay packets. Each node of the network should be given work on the basis of their residual energy.

- Avoid using the same node again and again for routing. This strategy can help the node to stay alive in the network. Most of the routing protocols choose the path with lesser number of hops. There is no concern for the residual energy of the nodes on that shorter path. So, there should be a trade-off between residual energy and number of hops while choosing a path to the destination node.

\section{LITERATURE REVIEW}

AODV (ad hoc on-demand distance vector routing protocol) [3] is designed to serve the purpose of routing of mobile nodes. It can route the packets in a network with large number of nodes. A routing table is maintained by AODV at each node containing parameters like Sequence number of destination, next hop, flags (routing, state). This makes it clear that information of the route from the source node to the destination node is clear and complete. Unlike DSR, in AODV there is a HELLO packet. DSR [4] works in two phases: route discovery and route maintenance. In the route discovery phase, source node broadcasts RREQ (route request) packet in the network. When a source node in a DSR sends a data packet, it completely defines the route from source to destination. DSR is not equipped with techniques to choose a route from multiple RREPs. This results in redundant and stale entries in cache of nodes in the network. This problem gets worse when nodes have high mobility. Figure 1 (a) shows the route discovery phase and figure 1 (b) shows route reply phase. Source broadcasts packet for requesting route to the destination in the network. Nodes $\mathrm{c}$ and $\mathrm{b}$ replies to the source node. Path 1-2 is chosen by the source node to transfer the packet to the destination.

DSDV (Distance Sequence Distance Vector) [18] routing protocol is proactive routing protocol. Table of routes is maintained with consistence and regularity. A single route is maintained to the destination. Although it provides paths 
which are loop-free but it does not utilize the bandwidth the table for route information but only to a smaller set of efficiently. Packets are broadcasted even when the routes nodes. Nodes are expected to have the knowledge of their are not changed. MANETs have limited bandwidth. So, neighboring nodes. In Zone Routing Protocol [19], nodes this routing protocol is not preferred. OLSR (Optimized of the network are divided into zones. Routing of packets Link State Routing Protocol) [19] is another proactive is done at two levels- locally at each node and globally at routing protocol. In this routing technique, re- the nodes of other zones. It is a reactive protocol when broadcasting of packets is done in a controlled manner dealing with inter-zone and proactive when dealing with with the assistance of neighboring nodes. Such intra-zone. Overlapping of zones can cause problem of neighboring nodes are called multi-point relay (MPR) inefficiency in this routing technique.

selector set. Nodes in the network chose their MPR. MPRs

can help the nodes of the network to reach other nodes and Most of the energy-aware routing protocols for MANET are updated with time. Although because of its architecture it can be used for a network without a centralized base station, but there is wastage of time and energy is discovering new paths when an existing path gets damaged or unresponsive.

TORA [18] is a hybrid routing protocol with high scalability. It works in three phase creation of routes, maintaining the route and removal of unwanted routes from the table. This routing protocol proactively creates

have chosen DSR and AODV as their base protocols because of their on-demand routing technique, which is suitable for a dynamic network. Also they do cause overhead of updating tables periodically. Bandwidth is efficiently utilized by these two. Some of the energyaware routing protocols based on DSR and AODV are reviewed in this paper. Hybrid routing protocols can also be used under special circumstances for a mobile network. They can be modified to perform efficiently with energyconservation of the network.

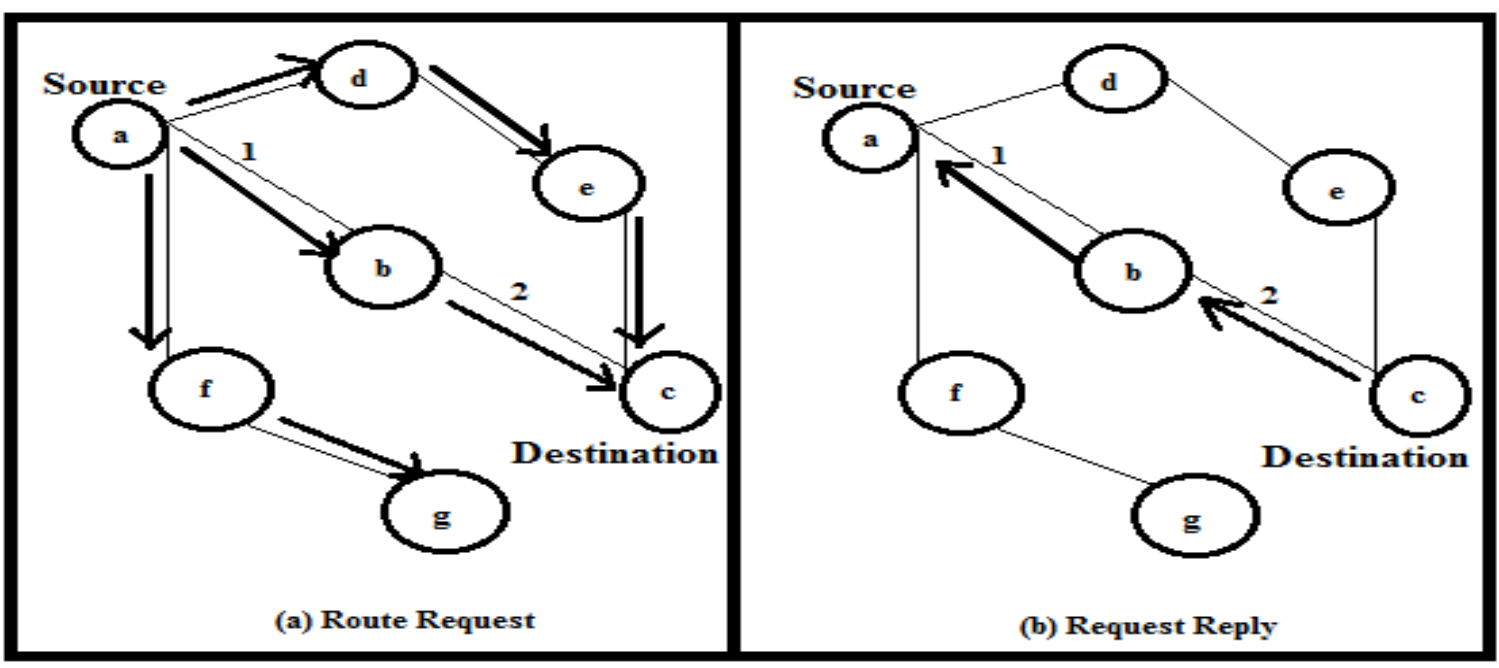

Figure 1 Dynamic Source Routing

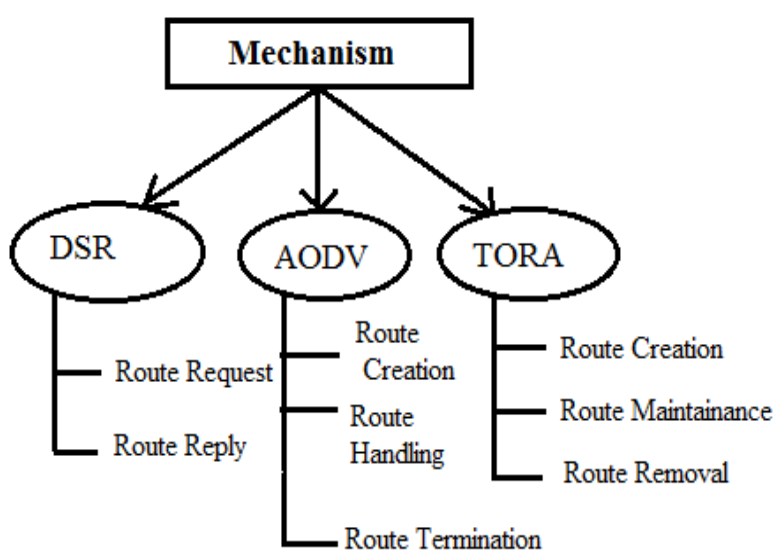

Figure 2 Phases of Routing Protocols named "rank" is calculated from current residual capacity, number of message transfers and internal load of the node in order to choose the best path. Those nodes which have balanced load and have sufficient residual capacity are chosen as intermediate nodes for the routing path. It provides power awareness to routing protocol. In [6], PDMRP shows that it is better than AODV as it also considers quality of services (QoS) like bandwidth and delay and satisfies them.

Paper in [7] proposed an energy efficient routing protocol (EERP) which helps to reduce the power of transmission of nodes in the network. RSS (received signal strength) technique is used for this purpose to find out the distance of one node from another, when data is being transmitted along a route. This value is then used to check which nodes are closer to the next hop.

A power aware routing scheme [5] considers internal load This algorithm modifies the three phases of AODV of nodes while choosing the routing path. A parameter protocol shown in fig. 2. Phases of other routing protocols 
Vol. 5, Issue 12, December 2016

are also shown in the respective figure. When using AODV as a routing protocol a route request (RREQ) packet is broadcasted. In highly dense networks it results into flooding of packets. A modified RREQ technique is proposed in [8] where RREQ are grid based. This approach improves the performance of AODV considerably by reducing the number of RREQ packets which are rebroadcasting. EDSR [9] modifies DSR by starting a timer whenever a RREQ is received by a node and the node adds its own cost to the path cost. Destination node sends RREP Intermediate nodes store the cost in their cache while hearing RREP. When a congested route is chosen in a MANET, it can lead to dropping of packets, delay in delivering packets and wastage of nodes' energy. A congestion aware routing protocol [10] is proposed to combat the problem of congestion. The work shows that it can perform better than DSR and uses two mechanisms to avoid congestion. Thus improves the lifetime of the network and increases its utilization.

Instead of using a single path, multiple paths are chosen in the technique proposed in [11]. Loads can be distributed by using the proposed technique and the network can have lesser number of packets dropped. Since multiple paths are chosen the algorithm is called multi-path dynamic source routing. One of the main issues of routing in Manet is limited power of nodes. PAMOR [12] (power aware multicast on-demand routing) not only utilizes the power efficiently but also utilizes bandwidth. Since this algorithm uses the approach of load balancing and dividing the traffic, it ensures security against attacks in the network.

Mobile ad hoc networks are of different types. One of such GAF [20] uses the concept of grid-positioning of nodes. type is metropolitan-area mobile ad hoc networks. A Location of nodes could be finding out by their fuzzy- based technique for routing is presented by coordinates based on grids and this helps to find out the Walaaet. al. Routing decisions are made locally at each representatives for rest of the nodes. Nodes with a limited node. This makes it a reactive routing protocol. Since battery are not considered as candidates for metropolitan-area mobile ad hoc networks are highly representatives. Grids communicate with the help of nodes dynamic, reactive routing protocols are suitable for it. with high energy in the respective grids.

TABLE 1: CATEGORIES OF ROUTING PROTOCOLS

\begin{tabular}{|l|l|l|}
\multicolumn{2}{|c}{ CATEGORIES } & \multicolumn{1}{c}{ EXAMPLES } \\
\hline \multirow{4}{*}{ A } & Reactive Routing Protocol & DSR, AODV \\
\cline { 2 - 3 } & Proactive Roiting Protocol & OLSR, DSDV \\
\cline { 2 - 3 } & Hybrid Routing Protocol & ZRP, TORA \\
\hline \multirow{3}{*}{ B } & Global Route Discovery & DSR, AODV \\
\cline { 2 - 3 } & Local Route Discovery & ZRP, PLR \\
\hline \multirow{3}{*}{ C } & Multipath Routing & RPBR, PDMRP \\
\cline { 2 - 3 } & Single path Routing & DSR, DSDV \\
\hline \multirow{2}{*}{ D } & Source Routing & DSR \\
\cline { 2 - 3 } & Hop-by-hop Routing & TORA, AODV \\
\hline
\end{tabular}




\section{IJARCCE}

Vol. 5, Issue 12, December 2016

\section{SUMMARY OF ENERGY AWARE ROUTING PROTOCOLS}

Existing routing protocols like DSR and AODV do not choose the routing path while considering the energy of nodes in the network. So energy aware routing protocols are the current need. There are several such protocols based on DSR and AODV. Such protocols are presented in this section in a tabularized form. Table 2 presents energy- efficient routing protocols which uses AODV as a base protocol. Energy can be conserved if certain modifications are done to existing routing protocols. DSR is also a good candidate for modifying so that routing is done with power-awareness. Several techniques which implements DSR with energy-efficiency are summarized in table 3. There are some other routing protocols which can be used for MANET, providing the benefits of increase in lifetime of the network. Table 4 presents such protocols in brief.

\section{TABLE 2: ENERGY EFFICIENT ROUTING PROTOCOLS BASED ON AODV}

\begin{tabular}{|c|c|c|c|}
\hline Proposed Algorithm & Year & Technique used & Advantage \\
\hline $\begin{array}{c}\text { Power Aware } \\
\text { Routing Scheme [5] }\end{array}$ & 2014 & $\begin{array}{c}\text { Calculate "rank" which is based on residual } \\
\text { capacity and load }\end{array}$ & $\begin{array}{c}\text { Internal load of nodes is } \\
\text { considered }\end{array}$ \\
\hline PDMRP [6] & 2014 & $\begin{array}{c}\text { Multi-paths are selected by considering } \\
\text { highest residual power and lesser hop-count }\end{array}$ & Quality of Services are satisfied \\
\hline EERP [7] & 2013 & RSS & $\begin{array}{c}\text { Transmission power of data } \\
\text { packets is reduced }\end{array}$ \\
\hline EGBB-AODV [8] & 2014 & Grid based broadcast of RREQ & $\begin{array}{c}\text { Decreases retransmitting of } \\
\text { RREQ, reduces flooding of } \\
\text { RREQ }\end{array}$ \\
\hline
\end{tabular}

TABLE 3: ENERGY EFFICIENT ROUTING PROTOCOLS BASED ON DSR

\begin{tabular}{|c|c|c|c|}
\hline Proposed Algorithm & Year & Technique used & Advantage \\
\hline EDSR[9] & 2013 & $\begin{array}{c}\text { Path costs are added to the RREQ and RREP, } \\
\text { each node adds its own cost to path cost }\end{array}$ & $\begin{array}{c}\text { Can find selfish nodes and } \\
\text { per packet energy } \\
\text { consumption is decreased }\end{array}$ \\
\hline CARM [10] & 2007 & $\begin{array}{c}\text { Higher throughput routes are selected, avoidance } \\
\text { of link data rates which are matched incorrectly. }\end{array}$ & Avoid congested links \\
\hline MDSR-NDR[11] & 2008 & $\begin{array}{c}\text { Cost function is dependent upon the load of each } \\
\text { path using complicated signaling mechanism }\end{array}$ & $\begin{array}{c}\text { Multi-path routing is } \\
\text { considered }\end{array}$ \\
\hline PAMOR [12] & 2010 & $\begin{array}{c}\text { Minimum energy of nodes is tracked while } \\
\text { sending RREQ and is used to }\end{array}$ & $\begin{array}{c}\text { Bandwidth utilization, } \\
\text { provides multicasting }\end{array}$ \\
\hline
\end{tabular}

TABLE 4: SOME OTHER ENERGY EFFICIENT ROUTING PROTOCOLS

\begin{tabular}{|c|c|c|c|}
\hline Proposed Algorithm & Year & Technique used & Advantage \\
\hline FRPM [13] & 2014 & $\begin{array}{c}\text { Fuzzy-based routing, decisions are made } \\
\text { locally, that means hop by hop }\end{array}$ & Easy to use and maintain \\
\hline RPBR [14] & 2013 & Uses packet holding time at each node & $\begin{array}{c}\text { Multi-path data transmission } \\
\text { balances load }\end{array}$ \\
\hline REMA [15] & 2015 & $\begin{array}{c}\text { REMAS, DMASFM, Data Forwarding and } \\
\text { maintenance of routes }\end{array}$ & $\begin{array}{c}\text { Outperforms existing } \\
\text { hierarchical routing protocols }\end{array}$ \\
\hline PLR [16] & 2001 & Local information present at the nodes & Cost and power efficiency \\
\hline GAF [20] & 2001 & $\begin{array}{c}\text { Nodes are in 3 states - active, discovery } \\
\text { and sleep. Master node is selected }\end{array}$ & $\begin{array}{c}\text { Recovers route preemptively, } \\
\text { power saving }\end{array}$ \\
\hline
\end{tabular}

\section{CONCLUSION AND FUTURE WORK}

Nodes in a MANET are highly mobile and have limited battery power. Reactive routing protocols like DSR, So they need to be modified and energy awareness in AODV, are preferred for routing data packets. But MANETs is the current need of the time. Our future work specifications of DSR and AODV are not sufficient for will be focused towards increasing the efficiency of routing of data packets in MANETs. They do not have any energy aware routing protocol and optimizing power mechanism to choose the route in an efficient way, for consumption of nodes in the network. 
Vol. 5, Issue 12, December 2016

\section{REFERENCES}

[1] Natesapillai, Karthikeyan, V. Palanisamy, and K. Duraiswamy. "A Review of Broadcasting Methods for Mobile Ad Hoc Network." International Journal of Advanced Computer Engineering, Serial Publications ISSN (2012): 0974-5785.

[2] Valikannu, R., A. George, and S. K. Srivatsa. "A novel energy consumption model using Residual Energy Based Mobile Agent selection scheme (REMA) in MANETs." Signal Processing and Integrated Networks (SPIN), 2015 2nd International Conference on. IEEE, 2015.

[3] Perkins, Charles, Elizabeth Belding-Royer, and Samir Das. Ad hoc on-demand distance vector (AODV) routing. No. RFC 3561. 2003.

[4] Johnson DB, Maltz DA (1996) Dynamic Source Routing in Ad Hoc Wireless Networks. In: Imielinski T, Korth H (eds) Mobile Computing, vol 353. Kluwer Academic Publishers, pp 153-181.

[5] Halder, TanmoyKanti, Chandreyee Chowdhury, and Sarmistha Neogy. "Power Aware AODV Routing Protocol for MANET." Advances in Computing and Communications (ICACC), 2014 Fourth International Conference on. IEEE, 2014.

[6] Othmen, Salwa, et al. "Power and Delay-aware Multi-Path Routing Protocol for Ad Hoc Networks." Computer, Information and Telecommunication Systems (CITS), 2014 International Conference on. IEEE, 2014.

[7] Bhatt, Uma Rathore, Paril Jain, and RakshaUpadhyay. "Enhanced AODV-An energy efficient routing protocol for MANET." Engineering (NUiCONE), 2013 Nirma University International Conference on. IEEE, 2013.

[8] Touzene, Abderezak, and Ishaq Al-Yahyai. "Performance Analysis of a Grid Based Route Discovery in AODV Routing Algorithm for MANET." Artificial Intelligence, Modelling and Simulation (AIMS), 2014 2nd International Conference on. IEEE, 2014. Varaprasad, Golla, and Suresh HosahalliNarayanagowda. "Implementing a new power aware routing algorithm based on existing dynamic source routing protocol for mobile ad hoc networks." Networks, IET 3.2 (2014): 137-142.

[9] Varaprasad, Golla, and Suresh HosahalliNarayanagowda. "Implementing a new power aware routing algorithm based on existing dynamic source routing protocol for mobile ad hoc networks." Networks, IET 3.2 (2014): 137-142.

[10] Chen, Xiaoqin, Haley M. Jones, and A. Dhammika S. Jayalath. "Congestion-aware routing protocol for mobile ad hoc networks." Vehicular Technology Conference, 2007. VTC-2007 Fall. 2007 IEEE 66th. IEEE, 2007.

[11] Tachtatzis, Christos, and David Harle. "Performance evaluation of multi-path and single-path routing protocols for mobile ad-hoc networks." Performance Evaluation of Computer and Telecommunication Systems, 2008. SPECTS 2008. International Symposium on. IEEE, 2008.

[12] Banerjee, Shilpa, et al. "A power aware multicast on-demand routing with load balancing." Computer Technology and Development (ICCTD), 2010 2nd International Conference on. IEEE, 2010.

[13] Gad, Walaa, and Tamer Abdelkader. "A fuzzy-based routing protocol for metropolitan-area mobile adhoc networks." Computer Engineering Conference (ICENCO), 2014 10th International. IEEE, 2014.

[14] Jung, Younho, et al. "A Residual Power Balancing Routing by Traffic-Splitting Transmission in Mobile Ad-Hoc Networks." Information Science and Applications (ICISA), 2013 International Conference on. IEEE, 2013.

[15] Valikannu, R., A. George, and S. K. Srivatsa. "A novel energy consumption model using Residual Energy Based Mobile Agent selection scheme (REMA) in MANETs." Signal Processing and Integrated Networks (SPIN), 2015 2nd International Conference on. IEEE, 2015.

[16] Stojmenovic I, Lin X. Power-aware localized routing in wireless networks. IEEE Transactions on Parallel and Distributed Systems 2001; 12(11): 1122-1133.

[17] Cho, Woncheol, and Seong-Lyun Kim. "A fully distributed routing algorithm for maximizing lifetime of a wireless ad hoc network." Mobile and Wireless Communications Network, 2002. 4th International Workshop on. IEEE, 2002.
[18] Bansal, Meenakshi, Rachna Rajput, and Gaurav Gupta. "Mobile ad hoc networking (MANET): Routing protocol performance issues and evaluation considerations." The internet society (1999).

[19] Z. J. Haas, M. R. Pearlman and P. Samar, "The Z one Routing Protocol (ZRP) for Ad Hoc Networks," draft- ietf-manet zone-zrp04.txt, work in progress, July 2008.

[20] YaXu, John Heidemann, and Deborah Estrin. "Geography-informed energy conservation for ad hoc routing," Proceedings of 7th Annual International Conference on Mobile Computing and Networking, pp. 70-84, July 2001. 